\section{Changes in antimicrobial prescribing behavior after the introduction of the antimi- crobial stewardship program: A pre- and post-intervention survey}

\author{
Ruchir Chavada, ${ }^{1}$ Harry N. Walker, ${ }^{2}$ \\ Deborah Tong, ${ }^{3}$ Amy Murray ${ }^{3}$ \\ ${ }^{1}$ Department of Microbiology and \\ Infectious Diseases, Pathology North \\ Central Coast, ${ }^{2}$ Division of Medicine, \\ Central Coast Local Health District; \\ ${ }^{3}$ Pharmacy Department, Central Coast \\ Local Health District, NSW, Australia
}

\section{Abstract \\ The introduction of an antimicrobial} stewardship (AMS) program is associated with a change in antimicrobial prescribing behavior. A proposed mechanism for this change is by impacting the prescribing etiquette described in qualitative studies. This study sought to detect a change in prescribing attitudes 12 months after the introduction of AMS and gauge utility of various AMS interventions. Surveys were distributed to doctors in two regional Australian hospitals on a convenience basis 6 months before, and 12 months after, the introduction of AMS. Agreement with 20 statements describing attitudes (cultural, behavioral and knowledge) towards antimicrobial prescribing was assessed on a 4-point Likert scale. Mean response scores were compared using the Wilcoxon Rank sum test. 155 responses were collected before the introduction of AMS, and 144 afterwards. After the introduction of AMS, an increase was observed in knowledge about available resources such as electronic decision support systems (EDSS) and therapeutic guidelines, with raised awareness about the support available through AMS rounds and the process to be followed when prescribing restricted antimicrobials. Additionally, doctors were less likely to rely on pharmacy to ascertain when an antimicrobial was restricted, depend on infectious diseases consultant advice and use past experience to guide antimicrobial prescribing. Responses to this survey indicate that positive changes to the antimicrobial prescribing etiquette may be achieved with the introduction of an AMS program. Use of EDSS and other resources such as evidence-based guidelines are perceived to be important to drive rational antimicrobial prescribing within AMS programs.

\section{Introduction}

Antimicrobial resistance (AMR) has been recognized as a major threat to global public health and collaborative multi-sectoral action is required to prevent a postantibiotic era. AMR is especially an issue in hospitals as patients with infections caused by resistant organisms have a longer length of hospital stay and greater morbidity and mortality. ${ }^{1}$ Antimicrobial stewardship (AMS) describes a suite of activities designed to maximize the rational use of antimicrobials and minimize the selection pressure for AMR. The introduction of AMS programs is associated with more rational antimicrobial prescribing patterns; however, initial decreases in the use of broad spectrum antimicrobials have been shown to regress to pre-AMS levels over months to years. ${ }^{1-3}$

A proposed mechanism for the initial change is via influencing the prescribing etiquette, described in qualitative studies of clinician attitudes toward antimicrobial prescribing. However, the effects of AMS programs on prescribing etiquettes of doctors are not well studied. ${ }^{4}$ Through defining the cultural and cognitive mechanisms of change in prescribing trends, AMS interventions may be developed to lengthen the duration of positive effects on prescribing behavior. The aim of this study was to identify any change in behavioral aspects to antimicrobial prescribing in the first 12 months after the introduction of AMS, and to gauge the usefulness of various interventions and resources adopted as a part of the AMS program.

\section{Materials and Methods}

AMS is now an integral part of Australian hospital accreditation standards. ${ }^{5}$ It was introduced on a formal basis in two hospitals in the Central Coast Local Health District (CCLHD) of New South Wales, Australia from March 2015. Of these, one hospital is a tertiary care hospital with subspecialty care and the other is secondary referral with general medicine and general surgery. A convenience sample of responses from doctors with varying levels of experience was gathered via an anonymous survey. Sampling was performed 6 months prior to the introduction of AMS and repeated with the same survey 12 months after the introduction of AMS.

With the introduction of the AMS program, antimicrobials were categorized into tiers of restriction using a traffic light system; green drugs were not routinely monitored, orange drugs required registration
Correspondence: Ruchir Chavada, Department of Microbiology and Infectious Diseases, Pathology North, Gosford Hospital, Gosford, NSW Australia.

E-mail: Ruchir.chavada@health.nsw.gov.au

Key words: Antimicrobial stewardship, AMS, behavior, interventions.

Acknowledgements: the authors would sincerely like to thank Lynn Francis, Biostatistician, University of Newcastle, NSW, Australia for the input into statistical analysis. We would also like to thank Dr Deo de Wit and Dr Gabrielle O'Kane for their input into the AMS program. We also appreciate the efforts of junior and senior medical staff who participated in the survey.

Contributions: the authors contributed equally

Conflict of interest: the authors declare no potential conflict of interest.

Received for publication: 16 June 2017

Revision received: 23 August 2017.

Accepted for publication: 25 August 2017.

This work is licensed under a Creative Commons Attribution-NonCommercial 4.0 International License (CC BY-NC 4.0).

(C) Copyright $R$. Chavada et al., 2017

Licensee PAGEPress, Italy

Infectious Disease Reports 2017; 9:7268

doi:10.4081/idr.2017.7268

and pre-prescription approval via an electronic decision support and approval system (Guidance MS®), Melbourne Health, Victoria, Australia), and red drugs required consultation with and approval by an Infectious Diseases physician or clinical microbiologist as well as registration on Guidance MS.

Antimicrobial stewardship team across both hospitals consists of 3 Infectious Diseases consultants with 2 full time AMS pharmacists. The structure of biweekly AMS ward rounds consists of intensive care antimicrobial stewardship round followed by similar rounds on patients in hospital wards on restricted antimicrobials. During these rounds there is an open discussion about the prescriptions of restricted antimicrobials with medical and surgical teams with documentation of suggestions made by the AMS team. The uptake of the AMS suggestions is followed up by the AMS teams at the next rounds. In cases where teams have not taken up the advise/suggestions or not complied, another discussion takes place with a more senior member (including the consultant involved in patient care) to 
understand the reason for the non-compliance. It has been observed that majority of the issues are resolved with these non-confrontational discussions.

Since introduction of AMS, there has been regular (6-12 monthly) feedback to medical/surgical teams and relevant departments about their antimicrobial prescribing. The feedback includes data which shows compliance with national, local or department specific guidelines, any side effects observed during treatment, microbiological results including local antibiograms, compliance with documentation of dose, duration and indication in medical notes/medication charts as well as reasons for noncompliance (if documented). The main idea of these feedbacks is to positively reinforce the culture of responsible prescribing, for doctors to feel confident in the microbiology results and to review their own prescribing practices. Besides this there are regular (every 3-6 monthly) education sessions given to the junior medical doctors where rational use of antimicrobials are discussed. Also regular audits assessing appropriateness of antimicrobial prescribing and compliance with guidelines are conducted every 12 months as part of National Antimicrobial Utilization Survey Program (NUASP), Australia. The results from NAUSP audits are systematically presented at hospital grand rounds every year.

The survey consisted of 25 questions which assessed the frequency of engaging in behaviors or holding attitudes relating to various aspects of antimicrobial prescribing (Appendix 1). While the survey questions were designed prior to AMS introduction in 2015 , some of the questions were adapted from the EPOC taxonomy. ${ }^{6}$ A recent Cochrane review by Davey et al classified behavioral interventions into 5 components; education, persuasion, restriction, environmental restructuring and enablement. ${ }^{7}$ In retrospect we think that some of the questions in the survey targeted these components.

Twenty responses were assessed on a 4point Likert-type scale, from never $=1$ to always $=4$. Six responses were yes-or-no in nature. Data on length of employment in the district, qualification level, and medical or surgical specialty of employment were also collected. Response scores for each question were compared using the Wilcoxon rank sum test, with statistical significance defined as $\mathrm{P}<0.05$.

\section{Results}

155 responses were collected before the introduction of AMS, and 144 afterwards.
Overall there were 42 consultant, 84 registrar and 173 resident and intern responses obtained during both surveys. The average length of employment within the hospital system was 1-2 years (IQR 6 months to $>5$ years). No significant differences were detected in characteristics between the preand post-intervention groups in terms of experience of the s doctors, their specialty or duration of employment (Table 1).

Table 2 shows the survey questions in order of presentation to respondents with demographic questions removed.

The following responses were noted; however were not statistically significant. Doctors seemed to have a greater appreciation for the importance of antimicrobial resistance in their patients (question 5) and relied more on the Australian antibiotic Therapeutic Guidelines and local specialty guidelines (question 6) after AMS was implemented. There was seemingly less reliance on pharmacist advice for antimicrobial prescribing (question 17), but greater likelihood for discussion within the team if there was any deviation from national guidelines (question 9). Doctors seemed more likely to check microbiology results within 48 hours of ordering the tests to assist with rationalization of antimicrobial therapy (question 10) and were more aware of when and how to seek approval for restricted antimicrobials (question 16).

On the other hand, the following responses were found to be statistically significant with a $\mathrm{z}$ value of $<1$. Doctors were less likely to rely on past or anecdotal prescribing experiences to guide antimicrobial prescribing after introduction of the AMS program (question $6, \mathrm{P}=0.02$ ). The differences in responses to questions $7(\mathrm{P}=0.01)$, $14 \quad(\mathrm{P}<0.001), 15 \quad(\mathrm{P}<0.001)$ and 21 $(\mathrm{P}=0.03)$ indicate that doctors readily

Table 1. Demographic characteristics of the survey respondents.

\begin{tabular}{lcc} 
Demographics & $\begin{array}{c}\text { Pre-AMS Survey } \\
(\mathrm{n}=\mathbf{1 5 5})\end{array}$ & $\begin{array}{c}\text { Post-AMS Survey } \\
(\mathrm{n}=144)\end{array}$ \\
Hospital A (Tertiary care) & 103 & 102 \\
Hospital B (Secondary referral) & 52 & 44 \\
\hline Designation of medical doctors & & 7 \\
$\quad$ Career medical officer (middle grade) & 8 & 21 \\
$\quad$ Consultant (senior grade) & 21 & 116 \\
$\quad$ Junior medical officer (junior grade) & 126 & 58 \\
Length of employment & & 44 \\
$0-12$ months & 52 & 18 \\
1-2 year & 51 & 24 \\
$2-5$ years & 31 & 69 \\
$>5$ years & 21 & 31 \\
Speciality & & 44 \\
Medical and related medical speciality & 55 & \\
Surgical and surgical subspecialties & 48 & \\
Emergency Medicine & 52 & \\
\hline
\end{tabular}

learned and adopted an EDSS for antimicrobial registration and also found it to be a useful resource for seeking guidance on prescribing and had a greater awareness and understanding of the process for prescribing restricted antimicrobials and the agents for which approval was required. Doctors developed independence in their operation of the EDSS and there was less reliance on pharmacists to remind them of which antimicrobials were restricted and thus required approval (question $21, \mathrm{P}=0.03$ ). 4) The AMS team were deemed readily accessible for advice after introduction of the program, (question 18, $\mathrm{P}=0.04$ ) .We also found that there were some responses with z $>1$ and which were also statistically significant. Doctors thought that receiving feedback about their team's antimicrobial prescribing practices (question 12, $\mathrm{P}<0.001$ ) and obtaining advice from the Infectious Diseases service was less useful (question $19, \mathrm{P}<0.001)$. There was also less need to rely on pharmacists reminding them of which antimicrobials were restricted and required approval (question 17, $\mathrm{P}<0.001$ ).

\section{Discussion}

It is important to understand the behavioral and attitude factors that play a role in antimicrobial prescribing in order to sustain efforts in AMS. A recent Cochrane review antimicrobial prescribing into five components; education (general education of prescribers), persuasion (increased level of communication), formulary restriction, environmental restructuring (increased use of point source tools like pocket guidelines and restricted antibiotic lists) and enablement (education with feedback). ${ }^{7}$ The categorized behavioral interventions in 
review concluded that enablement and restriction had the most marked and sustained behavioral effect on antimicrobial prescribing. The effect of feedback in addition to enablement and restriction was found to be moderate, while the effect of restriction to enablement was low. While enablement and restriction appear to be quite distinct in the mechanism of delivery of antimicrobial stewardship interventions, it does seem that there needs to be an element of both in AMS programs to achieve and maintain desired outcomes. In our hospitals we have implemented both with six monthly feedback to prescribers in the form of hospital grand rounds presentations and regular department-specific feedback, as well as ongoing formulary restriction of orange and red antimicrobials.

The implementation of regular auditing and feedback, EDSS and structured education as part of the AMS program was certainly appreciated in the results of our survey. We believe that these interventions empower prescribers and also allow them to retain the autonomy to prescribe antimicro- bials for patients admitted under their care. Behavioral change interventions in AMS have not been a part of the design and implementation of many studies in the literature. ${ }^{8}$ Understandably this makes the value of such interventions difficult to gauge. However, recently there is more data about the usefulness of AMS programs in which the use of EDSS results in favorable outcomes such as improvements in targeted antimicrobial use, reduced antimicrobial costs and length of hospital stay and decreased healthcare-associated Clostridium difficile infection rates. ${ }^{9}$ These results provide more substantiated evidence to clinicians that AMS programs lead to improvements in patient outcomes. As such, it may be valuable to provide ongoing feedback about reduction in healthcare-associated infections such as $C$. difficile, as well as trends in antimicrobial use and quality of antimicrobial prescribing. Interestingly, it was found in a study that AMS behavioral interventions were not a part of many professional society meetings, which may be considered a future target for AMS physi- cians and pharmacists. ${ }^{10}$

As with previous studies, we also found that the restrictive component of our AMS program was effective in helping to sustain continued rational antimicrobial prescribing. ${ }^{11}$ In the post-AMS implementation survey, more doctors were aware of the restricted list of antimicrobials and the process to undertake for seeking approval to use them. In a study by Tamma et al., postprescription review was found to be more useful in limiting days of therapy rather than pre-prescription authorization. However the authors conclude that both in tandem, as seen in our AMS program, would be more useful. ${ }^{12}$

Surprisingly, we found in the survey that after AMS introduction, doctors perceived receiving feedback about their team's prescribing behavior to be less useful. This effect may be due to junior doctors perceiving suboptimal feedback about their antimicrobial prescribing as detrimental on their reputation within the hospital, especially as seen by senior clinicians. There may also be an element of wishing to

Table 2. Survey questions with analysis of responses.

\begin{tabular}{|c|c|c|c|}
\hline No. & Question & $\mathbf{z}$ & $\mathbf{P}$ \\
\hline 5 & In my day-to-day practice I consider the impact of my antimicrobial prescribing on the emergence of resistance & -1.01 & 0.31 \\
\hline 6 & Indicate how frequently you use the various resources to guide your antimicrobial prescribing(see appendix 1 for details) & & \\
\hline $6 \mathrm{a}$ & Specialty/ward-specific guidelines (e.g. febrile neutropenia, sepsis in emergency department) & 0.59 & 0.56 \\
\hline $6 \mathrm{~b}$ & Reliance on local hospital guidelines & 1.17 & 0.24 \\
\hline $6 \mathrm{c}$ & Advice of those more senior and/or experienced in my team & 1.11 & 0.27 \\
\hline $6 \mathrm{~d}$ & Past clinical experience & 2.25 & $0.02 *$ \\
\hline $6 \mathrm{e}$ & Australian antibiotic (Therapeutic) Guidelines & 0.05 & 0.96 \\
\hline $6 \mathrm{f}$ & Pharmacist & -1.22 & 0.22 \\
\hline $6 g$ & Other guidelines & 1.24 & 0.22 \\
\hline 7 & There are sufficient resources, guidelines and support available in this hospital to drive rational and best practice antimicrobial prescribing & -2.49 & $0.01^{*}$ \\
\hline 8 & I question my team if an antimicrobial choice deviates from guidelines & -1.41 & 0.16 \\
\hline 9 & If my team prescribes an antimicrobial that deviates from guidelines I feel there is little I can do to change it & -0.11 & 0.91 \\
\hline 10 & I check pathology results within 48 hours of specimen collection to direct further antimicrobial therapy & -0.60 & 0.55 \\
\hline 11 & I am aware of when patients meet the criteria to switch from IV to oral antimicrobials & 1.61 & 0.11 \\
\hline 12 & It would be useful to receive feedback about my team's antimicrobial prescribing & 4.19 & $<0.001^{*}$ \\
\hline 13 & I am aware this hospital has restrictions on the use of some antimicrobials & 1.51 & 0.13 \\
\hline 14 & I know where to find a list of restricted antimicrobials for this hospital & -9.24 & $<0.001^{*}$ \\
\hline 15 & I know when I require approval for an antimicrobial I prescribe & -4.94 & $<0.001^{*}$ \\
\hline 16 & I know the process to obtain approval for an antimicrobial I prescribe & -0.99 & 0.32 \\
\hline 17 & I rely on pharmacists to advise me when approval is required for a restricted antimicrobial & 5.41 & $<0.001^{*}$ \\
\hline 18 & The Infectious Diseases service is easily contactable for advice and approvals & -2.06 & $0.040^{*}$ \\
\hline 19 & I am likely to rely on antimicrobial prescribing advice from the Infectious Diseases service & 3.53 & $<0.001^{*}$ \\
\hline 20 & I am likely to rely on antimicrobial prescribing advice from a pharmacist & 0.68 & 0.50 \\
\hline 21 & Using an electronic decision support and approval tool (e.g. Guidance MS) is useful to help guide antimicrobial therapy & -2.20 & $0.03^{*}$ \\
\hline 22 & I am aware there is an antimicrobial stewardship (AMS) team in CCLHD to optimise and support antimicrobial use & -5.27 & $<0.001^{*}$ \\
\hline 23 & I believe having an antimicrobial stewardship (AMS) program helps to improve patient care & 0.33 & 0.74 \\
\hline 24 & The benefits of antimicrobial stewardship (AMS) initiatives offset the changes to my workload & -1.07 & 0.29 \\
\hline
\end{tabular}


remain anonymous about departmental prescribing so as to appear in line with peers. While the exact reasons for this response are difficult to discern from a subjective survey, we believe that audit and feedback about antimicrobial prescribing is certainly useful and is supported by good evidence. ${ }^{7}$ Another unexpected observation was less reliance on advice from the Infectious Diseases service after implementation of the AMS program. This may possibly be explained by the way that the question was phrased, leading to differences in interpretation. The question may have been interpreted as asking how often a change in an antimicrobial prescription was made due to advice from Infectious Diseases/ Microbiology as opposed to any other reason for changing the prescription. If this was the case, decreased reliance on expert advice does not indicate decreased impact or value of the advice, but rather increased ownership and responsibility of the prescribing decision by the doctors themselves, using other resources like EDSS. This aspect would benefit from being investigated as a long term unintended consequence of AMS, which has not yet been described in other studies. A recent study observed that despite the introduction of an AMS program, there was no reduction in the number of Infectious Diseases consultations, and on the contrary, the authors saw a rise in consultations after AMS implementation. ${ }^{13}$ Whether corridor advice or curbside consultation made any impact on this particular question in our survey remains uncertain. Certainly more informal Infectious Diseases consultations have been received on AMS rounds as the consultants are easily accessible on the wards to discuss patients while doing AMS rounds. While an Australian study noted that a regression in compliance to AMS processes occurred several years after introduction, this effect would not have contributed so early on (12 months after introduction) in our AMS program. ${ }^{3}$ Several factors like drug shortages, changes in practices of individual units and antibiotic ballooning effect (use of another antibiotic class such as piperacillin-tazobactam instead of ceftriaxone) were thought to contribute towards this trend in that study. Our survey was not designed to investigate these factors as such trends are more important to measure and follow over a longer timeframe. This study has many limitations, and as like any other survey, the findings can only be considered descriptive and hypothesis-generating. The simultaneous testing of multiple hypotheses was not controlled for. The authors deemed excluding false positive findings less critical than avoiding type 2 statistical errors, in concor- dance with published recommendations. ${ }^{14}$ Data was unpaired, and a convenience method rather than a systematic method was employed for sampling. However, no statistically significant differences were observed between the samples in any of the demographic variables, providing some internal validity to the findings. The study was single-centered, as most doctors work in both participating hospitals, limiting the external validity of our findings. However, the components of the AMS program introduced in CCLHD are similar or identical to AMS programs in other Australian hospitals. ${ }^{2}$ Overall, these results suggest that doctors successfully learned and adapted to the processes required for our AMS program within 12 months. It also showed that AMS, at least in initial months, steered prescribers away from using past and anecdotal experience towards using electronic decision support tools to guide antimicrobial prescribing decisions. Further investigation into various interventions including a focus on audit and feedback would be helpful to analyze whether these measures are still the most sustainable and constructive strategies for shaping the attitudes and changing behavior for rational antimicrobial prescribing. The utility and acceptance of Infectious Diseases advice would also need to be monitored on an ongoing basis to understand how this changes in the context of established AMS programs.

\section{Conclusions}

Responses to this survey indicate that positive changes to the antimicrobial prescribing etiquette may be achieved with the introduction of an AMS program. Use of EDSS and other resources such as evidence-based guidelines appear to be important to drive rational antimicrobial prescribing within AMS programs.

\section{References}

1. de Kraker MEA, Davey PG, Grundmann H, et al. Mortality and hospital stay associated with resistant Staphylococcus aureus and Escherichia coli bacteremia: estimating the burden of antibiotic resistance in Europe. PLOS Med 2011;8:e1001104.

2. Buising KL, Thursky KA, Robertson $\mathrm{MB}$, et al Electronic antibiotic stewardship--reduced consumption of broadspectrum antibiotics using a computerized antimicrobial approval system in a hospital setting. J Antimicrob Chemother 2008;62:608-16.

3. Cairns KA, Jenney AW, Abbott IJ, et al.
Prescribing trends before and after implementation of an antimicrobial stewardship program. Med J Australia 2013;198:262-6.

4. Rattanaumpawan $\mathrm{P}$, Morales KH, Binkley S, et al. Impact of antimicrobial stewardship programme changes on unnecessary double anaerobic coverage therapy. J Antimicrob Chemother 2011;66:2655-8.

5. Australian Commission on Safety and Quality in Health Care. National Safety and Quality Health Service Standards (September 2012). Available from: https:/www.safetyandquality.gov.au/wp -content/uploads/2011/09/NSQHSStandards-Sept-2012.pdf.

6. Effective Practice and Organisation of Care. EPOC Taxonomy; 2015. Available frm: http://epoc.cochrane.org/ sites/epoc.cochrane.org/files/public/upl oads/epoc_taxonomy_13.12.16.pdf.

7. Davey P, Marwick CA, Scott CL, et al. Interventions to improve antibiotic prescribing practices for hospital inpatients. Cochrane Database Syst Rev 2017;2:CD003543.

8. Charani E, Castro-Sanchez E, Sevdalis $\mathrm{N}$, et al. Understanding the determinants of antimicrobial prescribing within hospitals: the role of "prescribing etiquette". Clin Infect Dis 2013;57:188-96.

9. Bond SE, Chubaty AJ, Adhikari S, et al. Outcomes of multisite antimicrobial stewardship programme implementation with a shared clinical decision support system. J Antimicrob Chemother 2017;72:2110-8.

10. Rawson TM, Moore LS, Tivey AM, et al. Behaviour change interventions to influence antimicrobial prescribing: a cross-sectional analysis of reports from UK state-of-the-art scientific conferences. Antimicrob Resist Infect Control 2017;6:11.

11. Charani E, Edwards R, Sevdalis N, et al. Behaviour change strategies to influence antimicrobial prescribing in acute care: a systematic review. Clin Infect Dis 2011;53:651-62.

12. Tamma PD, Avdic E, Keenan JF, et al What is the more effective antibiotic stewardship intervention: pre-prescription authorization or post-prescription review with feedback? Clin Infect Dis. 2016;64:537-43.

13. Messacar K, Campbell K, Pearce K, et al A Handshake from antimicrobial stewardship opens doors for infectious disease consultations. Clin Infect Dis 2017;64:1449-52.

14. Armstrong RA. When to use the Bonferroni correction. Ophthal Physiol Opt 2014;34:502-8. 